Communications in Physics, Vol. 24, No. 3S1 (2014), pp. 143-148

DOI:10.15625/0868-3166/24/3S1/5466

\title{
OPTICAL PROPERTIES OF SILICON NANOPARTICLES PREPARED BY LASER ABLATION METHOD IN VARIOUS MEDIA
}

\author{
CHU ANH TUAN \\ Institute of Materials Science, Vietnam Academy of Science and Technology \\ and \\ University of Engineering and Technology, Vietnam National University, Hanoi \\ NGUYEN THI THU TRANG, LE ANH TU, DUONG THI GIANG, PHAM HONG DUONG \\ Institute of Materials Science, Vietnam Academy of Science and Technology \\ PHAM THANH HUY \\ Advanced Institute for Science and Technology, Hanoi University of Science and Technology
}

E-mail: tuanca@ymail.com

Received 04 April 2014

Accepted for publication 24 May 2014

\begin{abstract}
Bright photoluminescent silicon nanoparticles were successfully fabricated from porous silicon target in air and n-hexane using $532 \mathrm{~nm}$ line of YAG:Nd pulsed laser. The whole procedure was carried out at room temperature, in atmosphere. The morphology and composition of as-synthesized nanoparticles were characterized by SEM and EDS measurements. Their optical properties were investigated. The PL peak position of the as-synthesized Si nanoparticle ablated in air is slightly blue-shifted in comparison with those ablated in n-hexane, while it was almost no change of peak position of Si nanoparticles fabricated from $p$-Si in hexane and original p-Si wafer target. In contrast to PL intensity quenching of original p-Si sample and particles produced in air, those produced in n-hexane solvent exhibited an enhancement. The presence of dangling bonds or energy transfer from excited Si particles to oxygen molecules on the surface can be the cause of the quenching effect.
\end{abstract}

Keywords: silicon nanoparticles, pulsed laser ablation, time-dependent photoluminescence spectra.

\section{INTRODUCTION}

Silicon nanoparticles have attracted much attention for their expectation of various applications such as solid state light emitting device [1], fluorescent biological labels [2,3], and chemical sensing [4]. Visible PL of Si particles can be observed when their size is below $3 \mathrm{~nm}$ and have adequate surface passivation. Up to now, different chemical and physical methods have been used to prepare the luminescent silicon nanoparticles including decomposition of disilane at high temperature [5], solution routes using a variety of reducing agents [6], microemulsion technique [7], synthesis in inverse micelles [8], and laser induced pyrolysis of silane [9]. For chemistry route, after synthesis of nano-sized Si powder, it is necessary to functionalize the surface of Si particle with organic chemicals such as alkoxysilanes [10], a mixture of xylene isomers, ethylbenzene, and 
isopropylbenzene [11] to get bright PL. Recently, pulsed laser ablation technique has emerged as a novel tool for pure nanofabrication [12]. However, in our limited knowledge, there are several groups working on synthesis of Si particles in various environments with this and similar method, the laser-fabricated Si nanoparticles show little or no visible PL [13,14]. Thus, to obtain bright $\mathrm{PL}, \mathrm{Li}$ and co-workers [15] carried out a two-step synthesis process. Whereas, first laser-driven $\mathrm{Si}$ powder was generated under specific pressure $(540 \mathrm{mbar})$ from silane followed by etching in mixture of $\mathrm{HF} / \mathrm{HNO}_{3}$ acid to reduce the particles size and passivate the particle surface with oxide. However, this method was dealing with silane, which is very dangerous chemical; furthermore, the reduction of Si nanocrystals size by etching process is not easy to control.

In this paper, we report on the results of luminescent Si nanoparticles fabrication in both air and in liquid environments using simple process. We started from luminescent porous silicon $(\mathrm{p}-\mathrm{Si}$ ) wafer prepared by conventional electro-chemical procedure. The preparation method and optical properties of p-Si have been studied and published elsewhere by our group [16] and other [17-19]. Then, Si nanoparticles can be produced in various environments without any specific conditions by laser ablation from p-Si substrate. In air, these nanoparticles were deposited on a transparent substrate to form a nanostructured film. Alternatively, these nanoparticles can be produced by laser ablation the p-Si substrate in various liquids, for example in hexane or in water to form luminescent colloids. Optical properties and morphology of as-prepared Si particles were studied and discussed.

\section{EXPERIMENTAL DETAILS}

Porous silicon samples were prepared by electrochemical etching processes (anodization) from p-type (100)-oriented silicon wafer (resistivity $0.5-3 \mathrm{ohm} . \mathrm{cm}$ ). Before the etching process, $0.1 \mu \mathrm{m}$ thick Al layers were deposited on the backsides of the wafer by using evaporation method. The anodization was performed in a $6 \% \mathrm{HF} / \mathrm{H}_{2} \mathrm{O}$ solution at room temperature using a Pt electrode. A $10 \mathrm{~mA} / \mathrm{cm}^{2}$ current density was applied for 30 minutes. The etched area of the sample was 1 $\mathrm{cm}^{2}$. The samples were rinsed with ethanol, dried under a stream of $\mathrm{N}_{2}$ and then stored at room temperature in air until the preparation of Si nanoparticles in the next step in various media.

For comparison purpose, an as-prepared $\mathrm{p}$-Si with the size of $10 \times 10 \mathrm{~mm}$ was cut into two $5 \times 10 \mathrm{~mm}$ pieces: one was ablated in the air environment and the other was used in liquid environment. The arrangement of the experimental setup is shown in Fig. 1. The second harmonic wavelength $532 \mathrm{~nm}$ of a YAG:Nd laser, with a pulse width of $30 \mathrm{ps}, 25 \mathrm{~mJ}$ max pulse energy, and $10 \mathrm{~Hz}$ repetition rate was used to generate nanoparticles from $\mathrm{p}$-Si wafers. In one experiment in air, p-Si wafer target was fixed on a transparent acrylic plastic plate $(30 \mathrm{~mm} \times 30 \mathrm{~mm})$ and then was irradiated for 20 minutes.

Under interaction between laser beam and p-Si material, Si nanoparticles can then be deposited on the transparent acrylic substrate. For generating nanoparticles in liquid media, p-Si was horizontally immersed in a glass cuvette $(10 \mathrm{~mm} \times 10 \mathrm{~mm})$ containing $500 \mu \mathrm{l}$-hexane solvent. Hexane solvent $(95 \%)$ was purchased from Xilong chemical Co., Ltd (China). The transparent acrylic plastic - pSi system and cuvette were then placed onto a moving stage which could be moved in the $\mathrm{x}, \mathrm{y}$ and $\mathrm{z}$ directions with accuracy of $\pm 0.5 \mu \mathrm{m}$. Pulse energy of $10 \mathrm{~mJ}$ were used, and the diameter of beam spot on the target is about $4 \mathrm{~mm}$. 

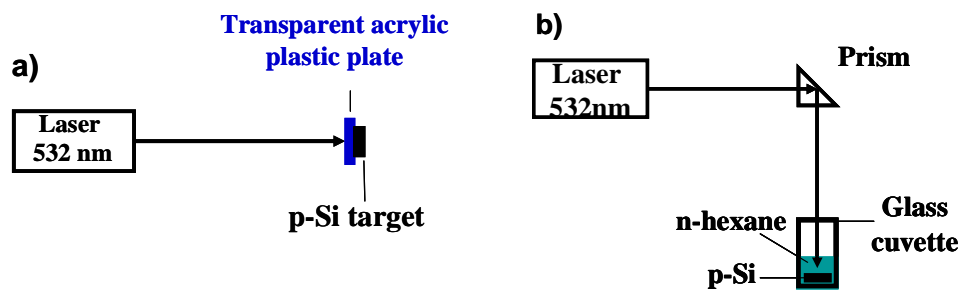

Fig. 1. Experimental set-up for Si nanoparticles preparation by laser ablation in air (a) and n-hexane (b).

Excitation and irradiation of the samples were realized with the $325 \mathrm{~nm}$ line of a He-Cd laser. The laser beam was focused with $40 \mathrm{~cm}$ focal length quartz lens to a spot of $\sim 400 \mu \mathrm{m}$ diameter. All the measurements were done in air at room temperature. All the recorded spectra were then corrected to the system sensitivity. The morphology and composition of the as-synthesized nanoparticles and the target wafers after the ablation process were fully characterized using the ?eld emission scanning electron microscope (FE-SEM) and energy-dispersive X-ray spectroscopy (EDS). FE-SEM and EDS analysis were performed on a JSM-7610F FEG-SEM at $100 \mathrm{kV}$.

\section{RESULTS AND DISCUSSION}

The morphology and composition of the laser - fabricated Si nanoparticles in air (a) and nhexane (b) solvent were revealed on the SEM images (left side) and corresponding EDS analysis (right side) in Figure 2, respectively. The SEM images clearly show that Si nanoparticles exhibit spherical-like geometry and were agglomerated. We observed the thin film not only on the surface of transparent acrylic substrate, but also on the bottom of cuvette after took p-Si wafer out of cuvette. It can be explained that, in air, as-prepared Si nanoparticles were ablated from p-Si wafer followed by deposition process to form thin film.

Whereas in $n$-hexane environment, these nanoparticles were unemployed chemical pretreatment process before performing SEM measurement resulting in agglutination on the bottom of cuvette forming nanostructured film. In the chemical treatment process, small amount of stabilizing chemicals are usually added to prevent agglomeration of the nanoparticles such as dodecanethiol [20]. Otherwise, the pulsed nanosecond laser ablation of a porous silicon wafer target in air tends to produce the size of particle smaller than that of Si nanoparticles generated in n-hexane medium. The range size is 10 to $23 \mathrm{~nm}$ for the Si powder nanoparticles produced in air and 15 to $50 \mathrm{~nm}$ for particles dispersion in n-hexane. This indicates that the laser interaction with the environment significantly affects on the nanocluster size after the ablation process. Under the laser plasma, the nanoparticles can be coagulated and formed much larger nanoscale spherical features.

Energy-dispersive X-ray spectroscopy (EDS) was used to determine the elemental composition of the particles. The EDS analysis shows peaks only for silicon and oxygen elements. The atomic percent concentration ratios of $\mathrm{Si}$ and $\mathrm{O}$ of the laser-fabricated particles in air and n-hexane are 27.4:52.1 and 36.3:36.5, respectively. These results confirm that the as-prepared nanoparticles are consistent with of $\mathrm{Si}$ inner/ $/ \mathrm{SiO}_{2}$ outer structure. The Si particles dispersion in 

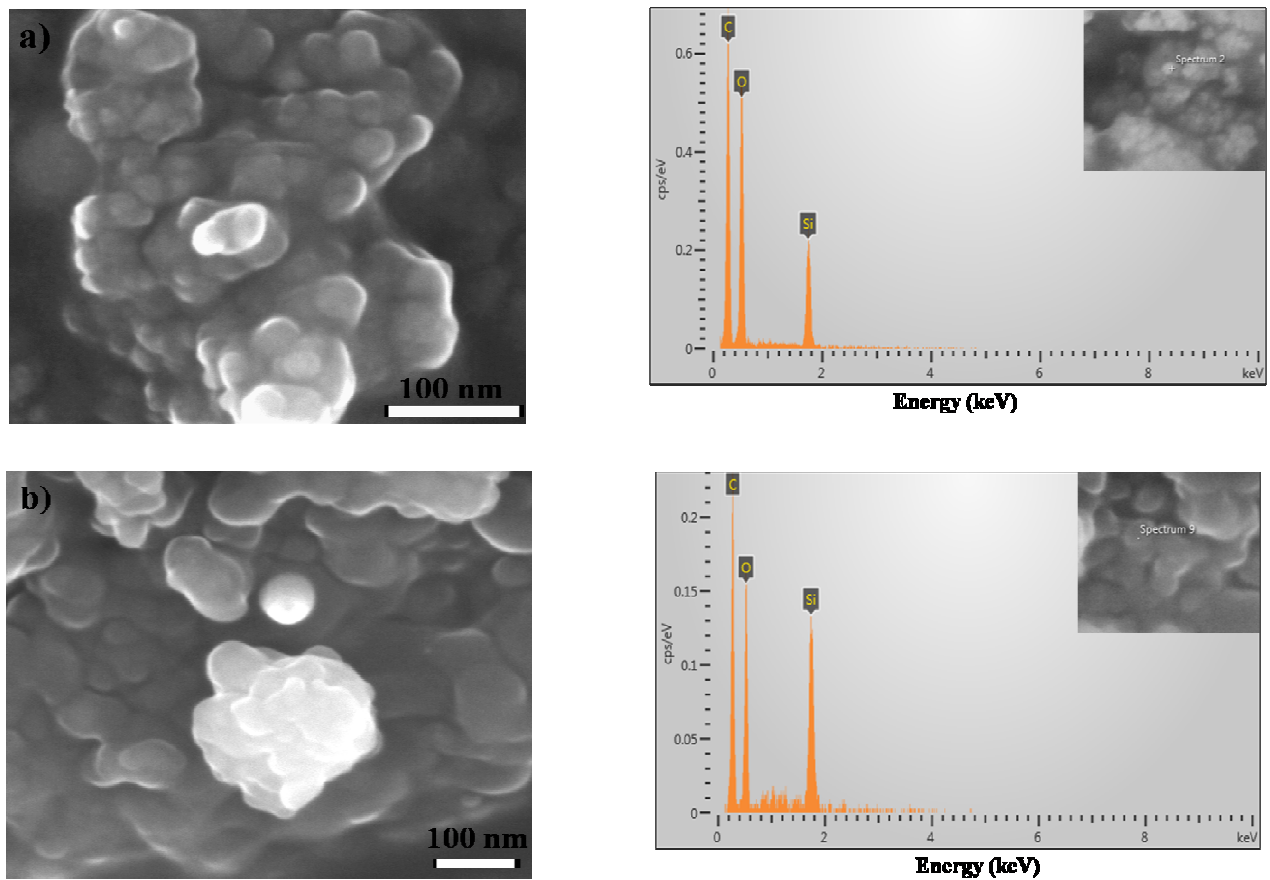

Fig. 2. Fig. 2a and $2 b$ show SEM images and corresponding EDS analysis of assynthesized Si nanoparticles prepared in air and n-hexane, respectively.

n-hexane solvent have a thinner layer of $\mathrm{SiO}_{2}$ than powder particles generated in air because $\mathrm{n}$ hexane solvent has a role in preventing particles from oxygen resulting in slow oxidation process of as-prepared Si particles.

The normalized photoluminescence spectra of the original p-Si specimen and the as-synthesized nanoparticles in air and n-hexane were shown in the Figure 3. The PL peak positions of these samples are observed at 682, 668.6 and $682 \mathrm{~nm}$, respectively, associated with Si-based nanostructures. It is noteworthy that the PL emission peak of the nanoparticle ablated in air is slightly blue-shifted compared with that of those ablated in n-hexane, while there was almost no change of peak position of Si nanoparticles fabricated from $\mathrm{p}-\mathrm{Si}$ in hexane and original $\mathrm{p}$-Si wafer target. These behaviors with bright visible PL of these particles were caused from the presentation of quantum confinement effect, in which, the PL depending on the size of particles. Therefore, the power particles will have the size smaller than that of particle fabricated in n-hexane solvent. In addition, the oxidation of Si nanoparticles in air much stronger than that in n-hexane, then, the size of pure Si particles performing in poor oxygen environment as n-hexane may be remained the size as original p-Si wafer target, and is bigger than that of powder particles in the same size. This result is consistent with the observation of particles size from SEM images.

To further investigate the optical properties of the as-prepared nanoparticles in air and $\mathrm{n}$-hexane environments at room temperature, we exposured $\mathrm{p}$-Si wafers under laser excitation $\lambda_{\text {exc }}=325 \mathrm{~nm}$, then measured continuously photoluminescence in exposured - time of $300 \mathrm{~s}$. 
The normalized PL intensity ratios depending on exposured - time of these samples are shown in Figure 4. For the powder particles (triangle line), the peak PL intensity decreased dramatically in the first 30 seconds during irritated by He-Cd laser, then achieved gradually stability. The reduction factor of the PL intensity is approximately 1.3. This behavior was also observed for the $\mathrm{p}-\mathrm{Si}$ wafer (circle line) with a reduction factor of about 1.8. The explanation for this phenomena was mentioned in the last our report [16]. Firstly, the dangling bonds on the surface will take place of $\mathrm{Si}-\mathrm{H}$ bonds during the irradiation of powder and original p-Si samples with $325 \mathrm{~nm}$ beam. Thus, the rise of non-radiactive recombination process via dangling bonds levels cause the quenching of PL intensity as shown in Fig. 4 ( $\circ$ line and $\nabla$ line). Secondly, the energy transfer from photo - excited nanoparticles to oxygen molecular was also a quenching channel. In constrict the peak PL intensity of Si particle prepared in n-hexane increase regularly during laser irradiation time. Otherwise, we also obtained that the PL intensity of these particles was stronger than that of those fabricated in air.

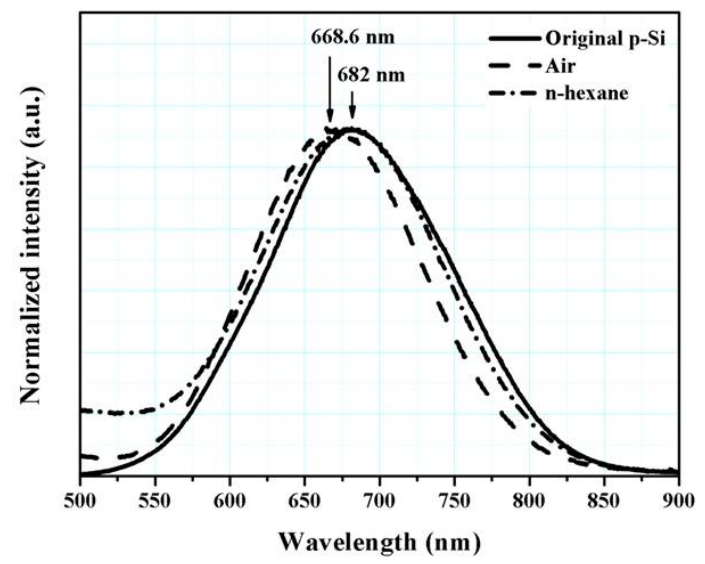

Fig. 3. The PL spectra of the original p-Si specimen (solid line) and the as-synthesized nanoparticles prepared in air (dash line) and n-hexane (dot dash line).

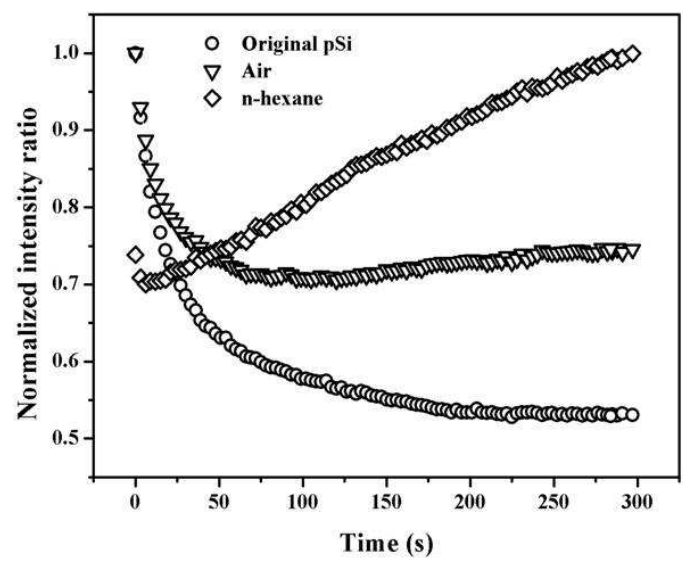

Fig. 4. Plot of normalized photoluminescence intensity ratio depending on exposured - time under laser excitation $\lambda_{\text {exc }}=325 \mathrm{~nm}$ of the original p$\mathrm{Si}$ wafer (o), powder $\mathrm{Si}$ prepared in air $(\nabla)$ and $\mathrm{Si}$ particle dispersion in n-hexane $(\diamond)$.

To explain the strange behavior of the change of PL intensity ratio depending on exposured - time, we collate our observation with the results obtained by Umezu et al. He reported on the stronger of the PL efficiency of particles dispersion in n-hexane comparing to that of pulsed laser ablation in background gases [14]. Based on the results shown in the FTIR spectra, he considered the $\mathrm{Si}-\left(\mathrm{CH}_{3}\right)_{n}$ bonds on the surface of $\mathrm{Si}$ nanoparticles as another factor contributing to the strong PL intensity of Si nanoparticles beside the quantum confinement effect. In our research, during irradiation time of Si nanoparticles dispersed in n-hexane solvent by laser, the hexane molecule decomposed continuously into $\mathrm{CH}_{3}$ species which passivity $\mathrm{Si}$ surface resulting in the increase of PL intensity. This character is possible for many applications, such as sensor and imaging. 


\section{CONCLUSIONS}

Si nanoparticles were fabricated successful by a simple pulsed laser ablation technique in air and n-hexane medium at room temperature. The as -prepared particles in n-hexane have the average size bigger than those prepared in air as seen in SEM images. The surface states and adsorbed species affect on the strong PL intensity of particles produced in both air and liquid environments. The passivity surface of Si nanoparticles produced in n-hexane by $\mathrm{CH}_{3}$ species causes the increase of the time-dependent PL intensity. While it is decrease for the as- prepared $\mathrm{Si}$ nanoparticles in air due to the replacement $\mathrm{S}-\mathrm{H}$ bonds by dangling bonds and the energy transfer from Si nanoparticles to oxygen molecular. This property has much potential in sensor and imaging applications.

\section{ACKNOWLEDGEMENTS}

The authors thank PhD. Luong Truc Quynh Ngan (IMS, VAST) for sharing expertise on preparation of p-Si wafer. This work was financially supported by the National Foundation for Science and Technology Development (NAFOSTED), Vietnam, under project No. 103.02.83.09.

\section{REFERENCES}

[1] R. Walters, H. Atwater, and G. Bourianoff, Nat. Mater., 4 (2005) 143.

[2] F. Erogbogbo, K. T. Yong, I. Roy, R. Hu, W. C. Law, W. Zhao, H. Ding, F. Wu, R. Kumar, M. T. Swihart, and P. N. Prasad, ACS Nano 5 (1) (2011) 413-423.

[3] Z. F. Li and E. Ruckenstein, Nano Lett. 4(8) (2004) 1463-1467.

[4] M. Sailor and E. Lee, Adv. Mater. 9 (1997) 783.

[5] L. E. Brus, P. F. Szajowski, W. L. Wilson, T. D. Harris, S. Schuppler, and P. H. Citrin, J. Am. Chem. Soc. 117 (1995) 2915-2922.

[6] D. Neiner, H. W. Chiu, and S. M. Kauzlarich, J. Am. Chem. Soc. 128 (2006) 11016-11017.

[7] R. D. Tilley and K. Yamamoto, Adv. Mater. 18 (2006) 2053-2056.

[8] J. H. Warner, A. Hoshino, K. Yamamoto, and R. D. Tilley, Angew. Chem. Int. Ed. 44 (2005) 4550-4554.

[9] F. J. Hua, M. T. Swihart, and E. Ruckenstein, Langmuir 21 (2005) 6054-6062.

[10] C. C. Tu, Q. Zhang, L. Y. Lin, and G. Cao, Opt. Exp. 20(S1) (2012) A69A74.

[11] K. Kusova, O. Cibulka, K. Dohnalova, I. Pelant, J. Valenta, A. Fucikova, K. Zidek, J. Lang, J. Englich, P. Matejka, P. Stepanek, and S. Bakardjieva, ACS. Nano 4(8) (2010) 4495-4504.

[12] A. V. Kabashin, M. Meunier, Recent Advances, in Laser Processing Material, Edited by J. Perriere, E. Millon, and E. Fogarassi, Elsevier, Amsterdam; pp. 1-36, 2006.

[13] N. G. Semaltianos, S. Logothetidis, W. Perrie, S. Romani, R. J. Potter, S. P. Edwardson, P. French, M. Sharp, G. Dearden, and K. G. Watkins, J. Nanopart. Res. 12 (2010) 573-580.

[14] I. Umezu, H. Minami, H. Senoo, and A. Sugimura, J. Phys.: Conf. Ser. 59 (2007) 392-395.

[15] X. Li, Y. He, S. S. Talukdar, and M. T. Swihart, Langmuir 19(20) (2003) 8490-8496.

[16] C. A. Tuan, T. T. Thuy, V. B. Nam, D. T. Cao, L. T. Ngan, P. H. Duong, and P. T. Huy, J. Sci. Tech. 50(1B) (2012) 398-404.

[17] S. Shih, K. H. Jung, J. Yan, D. L. Kwong, M. Kovar, J. M. White, T. George, and S. Kim, Appl. Phys. Lett. 63 (1993) 3306-3308.

[18] B. Huy, P. V. Hoi, P. H. Khoi, and N. T. Van, Int. J. Nanotachnol. 8(3/4/5) (2011) 360-370.

[19] L. Tsybeskov, J. V. Vandyshey, and P. M. Fauchet, Phys. Rev. B 49 (1994) 7821-7824.

[20] H. Anne, B. Stephan, and N. C. Boris, JLMN Journal of Laser Micro/Nanoengineering 3(2) (2008) 73-77. 